\title{
Stress relaxation of the infusion tube with a pressure sensor
}

\author{
Aoru Xie \\ Georgetown Preparatory School,Bethesda MD,US
}

\begin{abstract}
We studied stress relaxation of the infusion tube with a pressure sensor using FEA method. The stress distribution in the tube, the contact pressure on the wall of the pressure sensor, the decay of the reaction force on the wall of the pressure sensor were determined, respectively. Due to the stress relaxation of the infusion tube, the reaction force generated by the tube deformation was decreased by over $75 \%$ as compared with the original reaction force in the first several seconds after the infusion tube was set into the pressure sensor.
\end{abstract}

\section{Introduction}

An infusion pump is a medical device for delivering fluids into a patient's body in a controlled manner. There are many different types of infusion pumps for various purposes and under distinct conditions. Their safety feature significantly varies with the age and materials of the pumps. A "down pressure" sensor will detect when the patient's vein is blocked, or the line to the patient is kinked. This may be configurable for subcutaneous or venous applications. An "up pressure" sensor can detect when the bag or syringe is empty, or even if the bag or syringe is being squeezed. Reliability, sensitivity, and miniaturization are three critical factors to be considered when choosing force and pressure sensors for infusion pumps. Designers should consider pressure sensors that are fully compensated, amplified, and calibrated. Force and pressure sensors must provide high reliability and high sensitivity to ensure that the desired amount of medication is being delivered to the patient. Occlusion pressure, as an important parameter of infusion pumps, should be detected when an occlusion occurred.

Most of current infusion pumps have a preset occlusion pressure threshold. Once the pressure of the tubing which connects to the patient exceeds the preset limit, the occlusion alarm will be triggered. Some infusion pumps adopt a method of speed administration to trigger the occlusion alarm if the fluid rate stays zero for a certain time interval. However, the vascular pressure values are different in different individuals, or in the same person at different time points. Additionally, the alarm will not be activated if the pressure caused by an occlusion does not reach the preset threshold. In this case, the pump cannot function normally, and it will display erroneous values of the infusion rate and accumulated volume. These incorrect readouts could lead to catastrophic tissue or organ injuries in the patient. The higher the occlusion pressure is, the more serious the injury is. Therefore, the precise occlusion pressure detection is crucial for the performance of infusion pumps.

Despite the continuous efforts by healthcare practitioners, occlusions in central line catheters remain a significant issue which can result in delays in critical patient care, increased risk of infection, and increased healthcare costs. One important cause of catheter occlusion is blood reflux. Blood reflux can lead to thrombus formation which impedes the medicine infusion. An occluded catheter may also hamper blood withdrawal. Maintaining catheter patency and minimizing occlusions is important for enhancing patient safety and improve therapeutic outcomes.

\section{The Stress Relaxation Characteristics of Polyvinyl chloride}

Polyvinyl chloride (PVC) is a widely used material for intravenous infusion tubes and blood storage bags. Some PVC intravenous infusion tubes contain di-2-ethylhexyl phthalate (DEHP) as a plasticizer to provide flexibility of the infusion tube.

Stress relaxation is the decrease in stress in response to the same amount of strain generated in the structure. Stress relaxation describes how polymers relieve stress under constant strain. Because they are viscoelastic, polymers behave in a nonlinear, non-Hookean fashion. This non-linearity is described by both stress relaxation and a phenomenon known as creep, which describes how polymers strain under constant stress.

Viscoelastic materials have the properties of both viscous and elastic materials. They can be modeled by combining elements that represent these characteristics. One viscoelastic model called the Maxwell model predicts behavior akin to a spring (elastic element) being in series with a dash pot (viscous element), while the Voigt model places these elements in parallel. Although the Maxwell model is good at predicting stress relaxation, it is deficient in predicting creep. On the other hand, the 
Voigt model is good at predicting creep but not good at stress relaxation prediction.

In a one-dimensional relaxation test, the material is subjected to a sudden strain that is kept constant over the duration of the test, and the stress is measured over time. The initial stress is generated from the elastic response of the material. Then, the stress relaxes over time due to the viscous effects in the material. Typically, when a tensile, compressive, bulk compression, or shear strain is applied, the resultant data about stress vs. time can be fitted with a number of equations. Prony series, which contains multiple equations, can describe the static and dynamic features of viscoelastic materials.

In the infusion pump, the tube is compressed by the pressure sensor. When the fluid pressure in the tube rises, the reaction force generated by the pressure sensor will also rise. The total reaction force is affected by the fluid pressure and the force generated by the deformation of the tube. The force generate by the deformation of the tube will decrease because of the stress relaxation phenomenon.

\section{FEA method for stress relaxation analysis}

There are several types of FEA software which can simulate the structure of viscoelastic materials. The stress relaxation process can therefore be calculated and displayed by FEA software.

Complex inelastic behavior of materials includes hysteresis and viscoelasticity, according to the framework of finite strain viscoelasticity proposed by Simo. This framework is one of the most used model to interpret inelastic behavior due to its simplicity and easy implementation. Additionally, the effect of the strain-rate on the response is studied as well in Mullins effect, which is a phenomenon occurring in the first cycles of loading. Special attention has been paid to the influence of temperature and plastic behavior on large strains. The development of constitutive models which include these characteristics is not an easy task. Currently, complex material models based on continuum mechanics are trying to combine many of these characteristics. Good examples are recent models of viscoelasticity, plasticity and damages, as well as the thermo-mechanical models for large deformations.

Furthermore, additional research has focused on the analysis of the material behavior at different scales, leading to useful models for fiber-reinforced composites. Notably, the physically based models describes the complex rate, amplitude dependency, and rate and temperature dependency in a constitutive manner.

All these sophisticated models share some common features. Most of our work was conducted with Finite Element codes using a Lagrangian formulation, in which the internal variables were treated with special attention.

\section{Finite element simulation of stress relaxation}

\subsection{The structure}

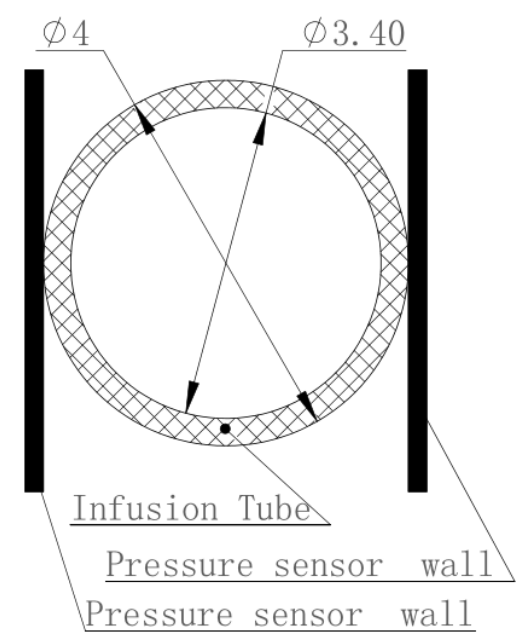

Figure 1. Schematic of the tube and the pressure sensor. The tube is not compressed in this figure. It will be compressed by the pressure sensor in the simulation process.

\subsection{Meshing settings}

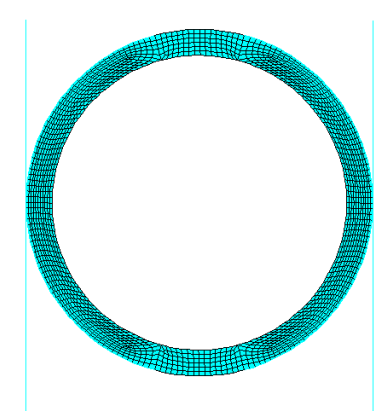

Figure 2. Structure of the mesh.

The vertical lines of on the left and right side represent the walls of the pressure sensor. The mesh size of the tube is $0.04 \mathrm{~mm}$.

\subsection{Loading settings}

In Figure 2, DOF of the left vertical line is set at 0 . The right vertical line will move to the left in 1 second. The reaction force generated by the deformation of the tube will be calculated in the following 10,000 seconds.

\subsection{Parameters of the material}



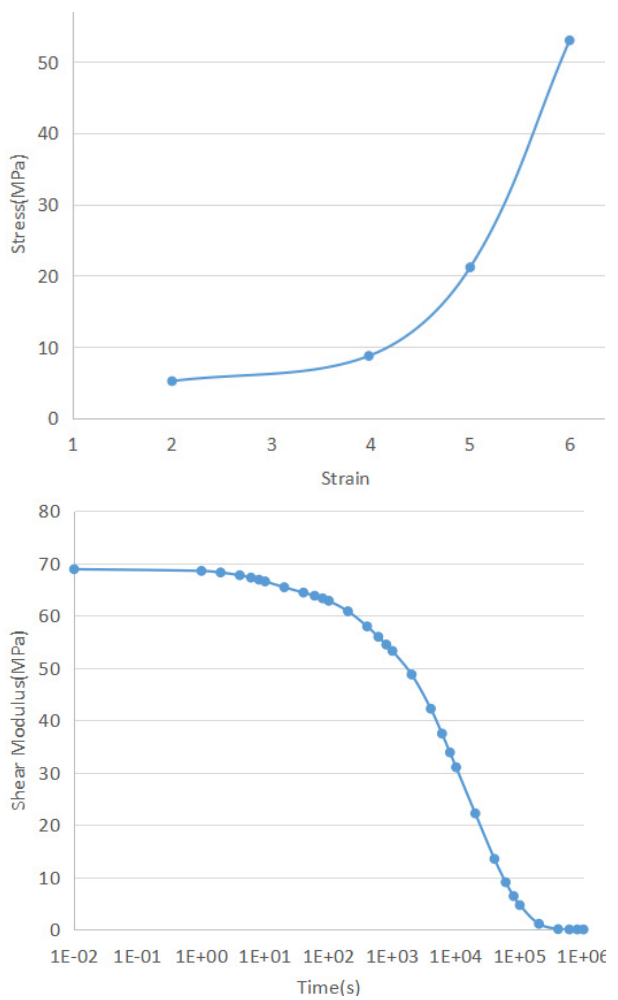

Figure 3. Parameters of the material.

Upper panel: Uniaxial Tension with Mooney 3 parameter. Lower panel: Shear modulus decay vs time.

\subsection{Solver settings}

Static analyse for the large deformation is conducted. In the simulation, the walls of the pressure sensor will contact the infusion tube. The contact pressure and the reaction force will be calculated.

\subsection{Results}

\subsubsection{Stress distribution}

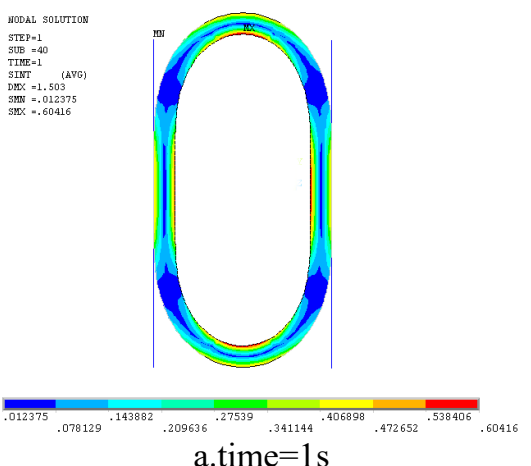

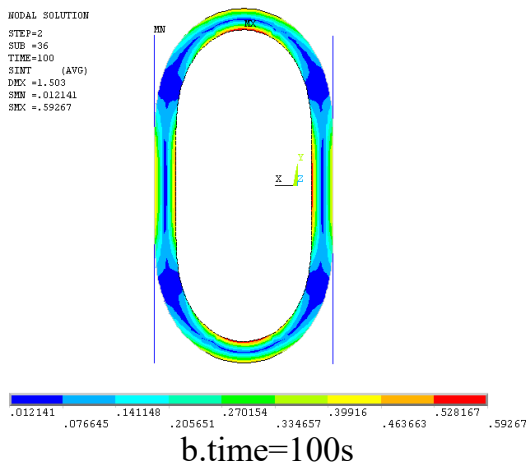

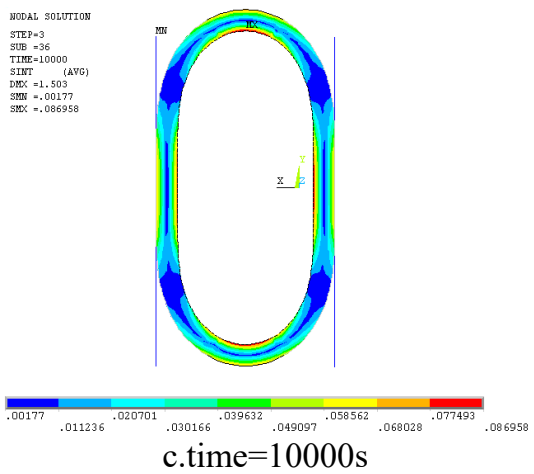

Figure 4. Stress distribution in each section of the tube during different time period.

The stress intense reduces as time lapses.

\subsubsection{Strain distribution}

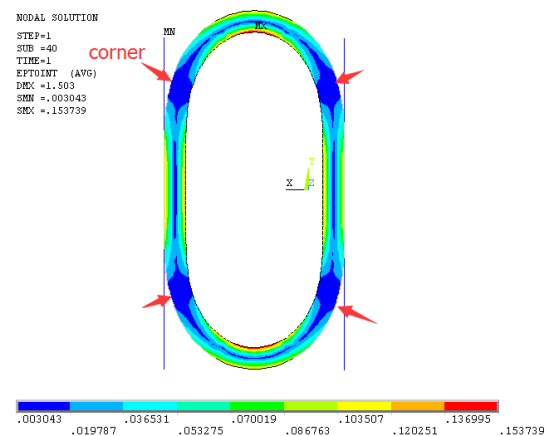

Figure 5. Strain distribution in each section of the tube. The strain distribution is consistent with the stress distribution. The four corners have very low strain intense.

4.6.3 Stress versus time at different locations of the tube 


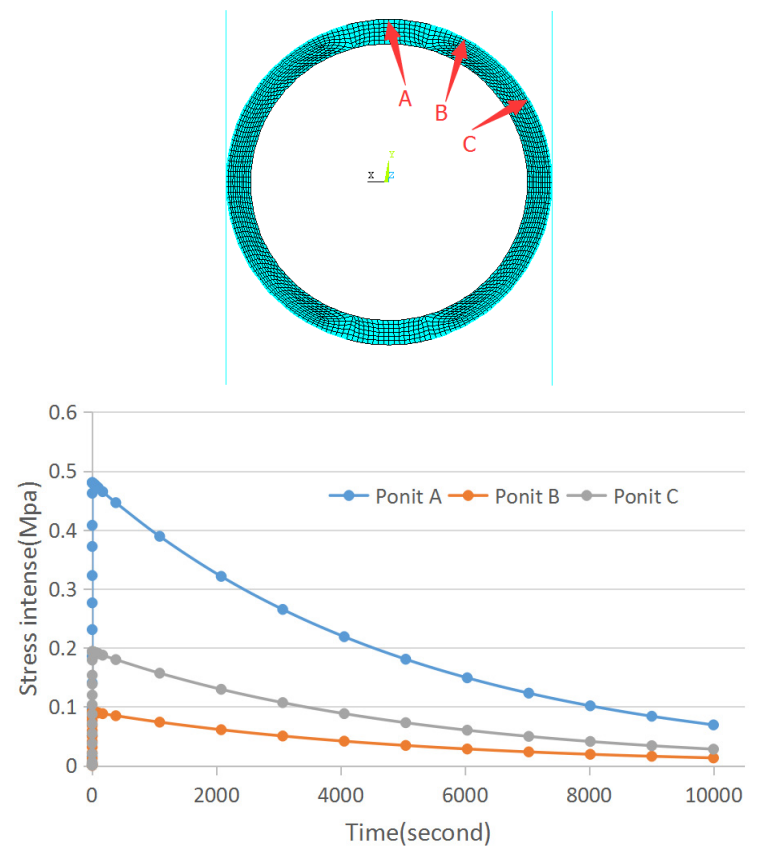

Figure 6. Stress vs time at different locations of the tube. Upper panel: Arrows show the chosen locations on the tube. Lower panel: Stress vs time at chosen locations of the tube. After 10,000 seconds, the stress intense is decreased by over $75 \%$ as compared with the stress intense at the first second.

\subsubsection{Pressure on the walls of the pressure sensor}
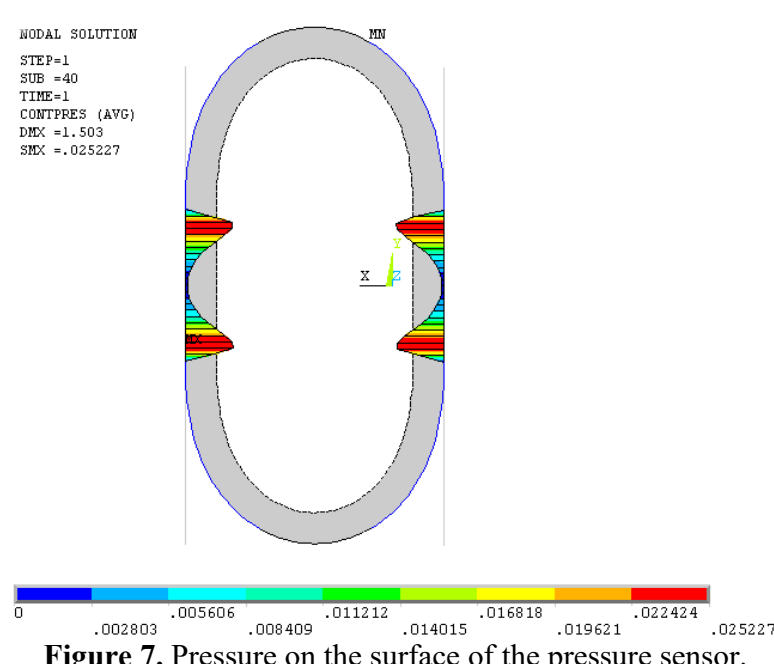

Figure 7. Pressure on the surface of the pressure sensor. The pressure on the walls of the sensor is uneven.

\subsubsection{Reaction force on the pressure sensor versus time}

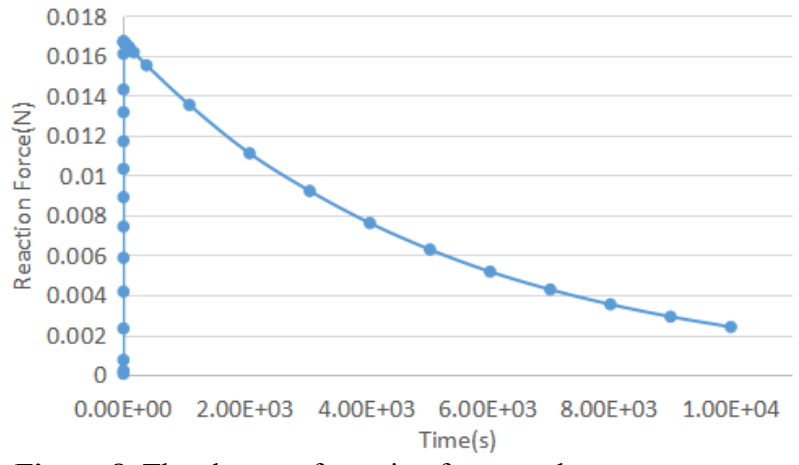

Figure 8. The change of reaction force on the pressure sensor over time.

After 10,000 seconds, the reaction force is decreased by more than $75 \%$ as compared with the stress intense at the start.

\subsubsection{Reaction force on the walls of the sensor vs} the width of the pressure sensor

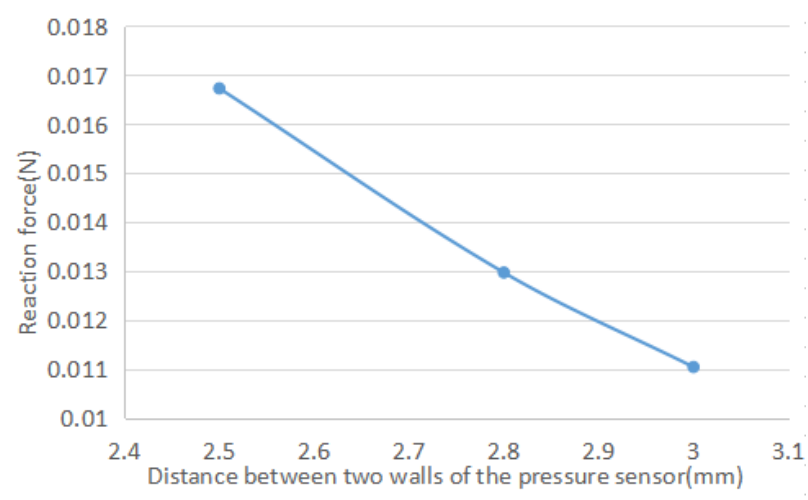

Figure 9. Reaction force on the surface of the sensor vs the width of the pressure.

The relationship between reaction force and the width of the pressure is almost linear.

\section{Conclusions and Prospects}

The reaction force on the walls of the pressure sensor decays over time, making it difficult to obtain the fluid pressure data in the tube. At different positions of the walls of the pressure sensor, reaction force generated by the deformation of the tube varies. Therefore, a better shape design of the walls of the sensor will facilitate the accurate reading of the fluid pressure in the infusion tube.

\section{Acknowledgement}

The author acknowledges the help from colleagues in the R\&D Department of Portable Medical Co. Ltd. This research is funded by Portable Medical Co. Ltd. The author also appreciates the support from the company.

\section{References}

1. Xiaoping Gao,Yize Sun,Zhuo Meng,Zhijun Sun. Viscoelastic Modeling of Yarn for Tufting Carpet. Procedia Engineering,10(2011) 
2. Long-Qiao Zhou,Sergey V. Meleshko. Group analysis of integro-differential equations describing stress relaxation behavior of one-dimensional viscoelastic materials. International Journal of NonLinear Mechanics, 77(2015)

3. Christian Huet. Extended Clapeyron formulae for viscoelasticity problems in the time domain and application to the boundary-condition effect in random composite bodies. Journal of the Mechanics and Physics of Solids,49(2001)

4. Kevin M. Labus,Christian M. Puttlitz. Viscoelasticity of brain corpus callosum in biaxial tension. Journal of the Mechanics and Physics of Solids,96(2016)

5. Rouhollah Alizadeh,James J. Beaudoin,Laila Raki. Viscoelastic nature of calcium silicate hydrate. Cement and Concrete Composites,32(2010)

6. Jiayue Shen,Peng Cheng,Wenting Gu,Zhili Hao. Stress relaxation measurement of viscoelastic materials using a polymer-based microfluidic device. Sensors \& Actuators: A. Physical,203(2013)

7. Kyle D. Allen,Kyriacos A. Athanasiou. Viscoelastic characterization of the porcine temporomandibular joint disc under unconfined compression. Journal of Biomechanics,39(2004)

8. J.T. Zhang,M. Zhang,S.X. Li,M.J. Pavier,D.J. Smith. Residual stresses created during curing of a polymer matrix composite using a viscoelastic model. Composites Science and Technology,130(2016)

9. Mohammad Hossien Saeidirad,Abbas Rohani,Saeed Zarifneshat. Predictions of viscoelastic behavior of pomegranate using artificial neural network and Maxwell model. Computers and Electronics in Agriculture,98(2013)

10. Christine E. Miller,Chandra L. Wong. Trabeculated embryonic myocardium shows rapid stress relaxation and non-quasi-linear viscoelastic behavior. Journal of Biomechanics,33(2000)

11. Georgia Dimitreli,Apostolos S. Thomareis. Texture evaluation of block-type processed cheese as a function of chemical composition and in relation to its apparent viscosity. Journal of Food Engineering,79(2006)

12. F.-J. Wortmann,K.V. Schulz. Non-linear viscoelastic performance of Nomex, Kevlar and polypropylene fibres in a single step stress relaxation test: 2. Moduli, viscosities and isochronal stress/strain curves. Polymer,36(1995)

13. Ville Tulkki,Timo Ikonen. Modelling anelastic contribution to nuclear fuel cladding creep and stress relaxation. Journal of Nuclear Materials,465(2015)

14. E. Sclarsky,J. Kadlowec,A.J. Vernengo. Modeling stress relaxation of crosslinked polymer networks for biomaterials applications: A distance learning module. Education for Chemical Engineers, 17(2016)

15. Ken Yamaguchi,Alan G. Thomas,James J.C. Busfield. Stress relaxation, creep and set recovery of elastomers. International Journal of Non-Linear Mechanics,68(2015)

16. Minwu Yao,Gareth H. McKinley,Benoit Debbaut. Extensional deformation, stress relaxation and necking failure of viscoelastic filaments 1 Dedicated to Professor Marcel J. Crochet on the occasion of his 60 th birthday. 1. Journal of Non-Newtonian Fluid Mechanics,79(1998)

17. M. Heuchel,J. Cui,K. Kratz,H. Kosmella,A. Lendlein. Relaxation based modeling of tunable shape recovery kinetics observed under isothermal conditions for amorphous shape-memory polymers. Polymer,51(2010)

18. Ratchada Sopakayang,Raffaella De Vita,Albert Kwansa,Joseph W. Freeman. Elastic and viscoelastic properties of a type I collagen fiber. Journal of Theoretical Biology,293(2012)

19. T.M. Atanackovic,S. Pilipovic,D. Zorica. Distributed-order fractional wave equation on a finite domain. Stress relaxation in a rod. International Journal of Engineering Science,49(2010)

20. Stephen H. Spiegelberg,Gareth H. McKinley. Stress relaxation and elastic decohesion of viscoelastic polymer solutions in extensional flow. Journal of Non-Newtonian Fluid Mechanics,67(1996)

21. C. Machiraju,A.-V. Phan,A.W. Pearsall,S. Madanagopal. Viscoelastic studies of human subscapularis tendon: Relaxation test and a Wiechert model. Computer Methods and Programs in Biomedicine,83(2006) 